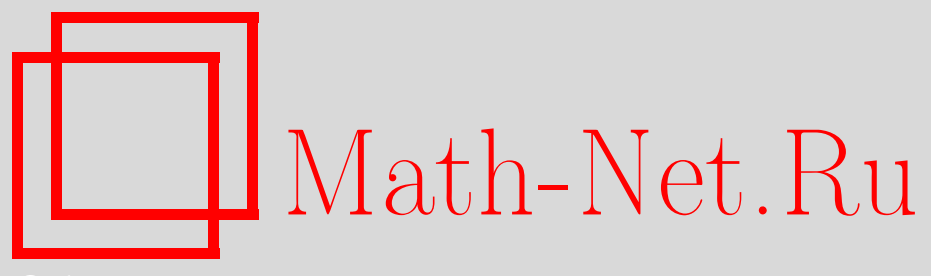

М. Бакурадзе, Формальные группы Бухштабера, Кричевера и Надирадзе совпадают, УМH, 2013, том 68, выпуск 3, 189-190

DOI: https://doi.org/10.4213/rm9521

Использование Общероссийского математического портала Math-Net.Ru подразумевает, что вы прочитали и согласны с пользовательским соглашением http://www . mathnet.ru/rus/agreement

Параметры загрузки:

IP : 52.87 .193 .239

26 апреля 2023 г., 14:54:50

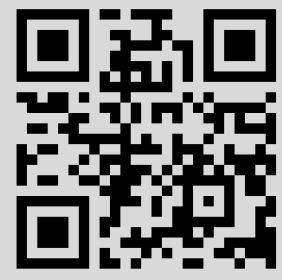




\section{Формальные группы Бухштабера, Кричевера и Надирадзе совпадают}

\section{М. Бакурадзе}

Пусть $F(x, y)$ - формальная группа геометрических кобордизмов [6]. Следуя Квиллену [7], отождествим ее с универсальной формальной группой Лазара. Положим $\omega(x)=\frac{\partial F(x, y)}{\partial y}(x, 0)$.

В [2] В. М. Бухштабер дает аналитическое решение функционального уравнения на экспоненту формальной группы вида

$$
\mathscr{F}_{\mathrm{B}}(x, y)=\sum \alpha_{i j} x^{i} y^{j}=\frac{A(y) x^{2}-A(x) y^{2}}{B(y) x-B(x) y} .
$$

Лемма 1. Пусть $\mathscr{F}$ - формальная группа вида $\mathscr{F}$ В, где $B^{\prime}(0)=A^{\prime}(0)$. Тогда $B(x)$ совпадает с ограничением $\omega(x)$, т.е. $B(x)$ является образом $\omega(x)$ при кольцевом гомоморфизме кольца Лазара, классифицирующем формальную группу Ғ․

Действительно, если ряды $A$ и $B$ имеют вид $A(t)=A_{0}+A_{1} t+A_{2} t^{2}+O\left(t^{3}\right)$ и $B(t)=B_{0}+B_{1} t+B_{2} t^{2}+O\left(t^{3}\right)$, то $\mathscr{F}(x, y)=\left(A_{0} / B_{0}\right)(x+y)+O(x y)$. Таким образом, если $\mathscr{F}(x, y)$ является формальной группой, то должно выполняться равенство $A_{0}=B_{0}$ и после соответствующего деления числителя и знаменателя можно считать, что $A_{0}=B_{0}=1$. Далее, $\mathscr{F}(x, y)=x+y+A_{1} x y+\sum_{i=2}^{\infty} B_{i}\left(x^{i} y+x y^{i}\right)+O\left(x^{2} y^{2}\right)$. Таким образом, $\alpha_{1 i}=B_{i}$ при $i \geqslant 2, \alpha_{11}=A_{1}=A^{\prime}(0)=B^{\prime}(0)$ и ряд $1+\sum_{i \geqslant 1} \alpha_{1 i} t^{i}$ совпадает с ограничением формы $\left(1+\sum_{i \geqslant 1}\left[\mathbb{C} P^{i}\right] t^{i}\right)^{-1}=\omega(t)$.

Теперь внесем небольшие изменения в исследование формальной группы $\mathscr{F}$ В, проведенное в [5], и, как подсказывает лемма 1, введем

$$
A(x, y)=\sum A_{i j} x^{i} y^{j}=F(x, y)(x \omega(y)-y \omega(x)) .
$$

Определим универсальный формальный групповой закон Надирадзе $\mathscr{F}_{\mathrm{N}}$ с помощью очевидного классифицирующего отображения кольца Лазара на его факторкольцо по идеалу, порожденному всеми $A_{i j}, i, j \geqslant 3$.

ПредЛОЖЕНИЕ 2. Пусть $L-$ кольцо Лазара. Тогда:

i) $\omega^{\prime}(x)-\omega^{\prime}(0)=2 x \widehat{\omega}(x)$ в $L[[x]]$, где $\widehat{\omega}(x)=\sum_{i \geqslant 1} \omega_{i} x^{i-1}$;

ii) в $L[[x, y]] /(x y)^{3}$ имеем

$$
A(x, y)=\left(x \omega(y)+y \omega(x)-\omega^{\prime}(0) x y\right)(x \omega(y)-y \omega(x))+(\omega(x) \widehat{\omega}(x)-\omega(y) \widehat{\omega}(y)) x^{2} y^{2} .
$$

ДоказАтЕЛЬСтво. Пусть $f$ и $g$-экспонента и логарифм формального группового закона $F$. Тогда $F(x, y)=f(g(x)+g(y))$ и

$$
f^{\prime}(x)=1 / g^{\prime}(f(x))=\omega(f(x)), \quad f^{\prime}(g(x))=\omega(f(g(x)))=\omega(x) .
$$

Пусть $\omega(x)=1+b_{1} x+b_{2} x^{2}+\cdots$. Поскольку $g^{\prime \prime}(0)=-f^{\prime \prime}(0)=-\omega^{\prime}(0)=-b_{1}$, то

$$
\frac{\partial^{2} F}{\partial y^{2}}(x, 0)=f^{\prime \prime}(g(x))+f^{\prime}(g(x)) g^{\prime \prime}(0)=\omega^{\prime}(x) \omega(x)-\omega^{\prime}(0) \omega(x) .
$$

Отсюда получаем i), так как правая часть (4) делится на 2 и $\omega(x)$ обратима. ii) Ввиду антисимметрии по модулю $(x y)^{3}$ имеем $A(x, y)=A(y) x^{2}-A(x) y^{2}=\sum\left(A_{i 2} x^{2} y^{i}-\right.$ $\left.A_{i 2} x^{i} y^{2}\right)$. Мы хотим вычислить $-\sum A_{i 2} x^{i}$ в терминах $\omega(x)$. Применив $\frac{\partial^{2}}{\partial y^{2}}(x, 0)$ к $(2)$

Работа выполнена при поддержке Volkswagen Foundation, Ref.: I/84 328.

DOI: $10.4213 / \mathrm{rm} 9521$ 
и учитывая (3) и (4), получим $-2 \sum A_{i 2} x^{i}=x \omega(x) \omega^{\prime}(x)-x \omega^{\prime}(0) \omega(x)+2 x \omega^{\prime}(0) \omega(x)-$ $2 \omega^{2}(x)+2 b_{2} x^{2}$. Так как коэффициенты лежат в кольце Лазара, отсюда следует равенство $-\sum A_{i 2} x^{i}=x \omega(x)\left(\omega^{\prime}(x)-\omega^{\prime}(0)\right) / 2+x \omega^{\prime}(0) \omega(x)-\omega^{2}(x)+b_{2} x^{2}$, поэтому

$$
\begin{aligned}
& \sum(\left(A_{i 2} x^{2} y^{i}-A_{i 2} x^{i} y^{2}\right)=(x \omega(y)+y \omega(x))(x \omega(y)-y \omega(x)) \\
&-\omega^{\prime}(0) x y(x \omega(y)-y \omega(x))+\omega(x) \widehat{\omega}(x) x^{2} y^{2}-\omega(y) \widehat{\omega}(y) x^{2} y^{2} .
\end{aligned}
$$

Предложение доказано.

Для вычисления рода Кричевера на коэффициентах формальной группы геометрических кобордизмов в [3] универсальная формальная группа Кричевера $\mathscr{F}_{\text {Kr }}$ определена как

$$
\mathscr{F}_{\mathrm{Kr}}(x, y)=x b(y)+y b(x)-b^{\prime}(0) x y+\frac{b(x) \beta(x)-b(y) \beta(y)}{x b(y)-y b(x)} x^{2} y^{2},
$$

где $\beta(x)=\frac{b^{\prime}(x)-b^{\prime}(0)}{2 x}$. В [3] также показано, что $b(x)=\frac{\partial \mathscr{F} \mathrm{Kr}}{\partial y}(x, 0)$. Из леммы 1 и предложения 2 , ii) следует, что $\mathscr{F}_{\mathrm{Kr}}$ также может быть определена с помощью классифицирующего отображения формальной группы $\mathscr{F}_{\mathrm{N}}$, если в качестве $b(x)$ взять ограничение инвариантной формы $\omega(x)$. Таким образом, $\mathscr{F}_{\mathrm{N}}=\mathscr{F}_{\mathrm{Kr}}$.

Следуя [2], авторы работы [4] рассматривают следующую формальную группу, соответствующую роду Кричевера $\mathscr{K} v$ :

$$
\mathscr{F}_{\mathscr{B}}\left(u_{1}, u_{2}\right)=u_{1} c\left(u_{2}\right)+u_{2} c\left(u_{1}\right)-a u_{1} u_{2}-\frac{d\left(u_{1}\right)-d\left(u_{2}\right)}{u_{1} c\left(u_{2}\right)-u_{2} c\left(u_{1}\right)} u_{1}^{2} u_{2}^{2} .
$$

Из леммы 1 и предложения 2, ii) следует, что (при $\left.c^{\prime}(0)=a\right)$ ряд $\mathscr{F}_{\mathscr{B}}(x, y)$ совпадает с $(5)$, т. е. $c(x)=b(x)$ и $d(x)=-b(x) \beta(x)$.

СлеДСТвиЕ 3. Верны равенства $\mathscr{F}_{\mathrm{N}}=\mathscr{F}_{\mathrm{Kr}}=\mathscr{F}_{\mathscr{B}}$, причем кольцо коэффициентов является фактором кольиа Лазара по идеалу, порожденному всеми $A_{i j}, i, j \geqslant 3$.

В [1] при помощи MAPLE вычислено кольцо коэффициентов формального группового закона Надирадзе $\mathscr{F} \mathrm{N}$ до размерности 26. А именно, найден набор полиномиальных образующих $z_{1}, z_{2}, \ldots$ кольца Лазара, для которых соотношения малых размерностей имеют вид $5 z_{5}=z_{2} z_{3}+2 z_{1} z_{4}, 2 z_{6}=0, z_{1} z_{6}=0, z_{3} z_{6}=0, z_{10}=0, z_{5} z_{6}=0$, $z_{12}=0$, а элементы $7 z_{7}, 2 z_{8}, 3 z_{9}, 11 z_{11}$ и $13 z_{13}$ разложимы. Заметим, что наши вычисления согласуются с результатами работы [3] о структуре кольца коэффициентов формальной группы $\mathscr{F}_{\mathrm{Kr}}$, полученными в терминах уравнения ассоциативности. Здесь новой информацией о группе (а следовательно, о роде) Кричевера, является то, что поскольку $z_{10}=0, z_{12}=0$, то в размерностях 20 и 24 нет неразложимых элементов. Возникает вопрос, в каких размерностях каждый элемент мультипликативно разложим.

\section{Список литературы}

[1] M. Bakuradze, M. Jibladze, Proc. A. Razmadze Math. Inst., 159 (2012), 1-9. [2] B. M. Бyxштабер, УМН, 45:3(273) (1990), 185-186. [3] В. М. Бухштабер, Е. Ю. Бунькова, Функц. анализ и его прил., 45:2 (2011), 23-44. [4] V. Buchstaber, T. Panov, N. Ray, Int. Math. Res. Not. IMRN, 2010, № 16, 3207-3262. [5] R. Nadiradze, Formal group and cohomology theories, Dissertation for the Doct. of Sci. Degree, Tbilisi, 1995. [6] С. П. Новиков, Изв. АН СССР. Сер. матем., 31:4 (1967), 855-951. [7] D. Quillen, Bull. Amer. Math. Soc., 75:6 (1969), 1293-1298.

\section{М. Бакурадзе (M. Bakuradze)}

Тбилисский государственный университет им. Ив. Джавахишвили, Грузия

E-mail: malkhaz.bakuradze@tsu.ge
Представлено В. М. Бухштабером Принято редколлегией 08.04.2013 